\title{
Correspondence
}

Editor: Ian Pullen

Contents: The Camberwell collaborative depression study/Obsessive-compulsive rituals/Near-death experience/Teenage depressive stupor/Defining personality disorder/Positive symptoms of schizophrenia/Internal monitor defect in schizophrenia/ Naltrexone and clonidine in heroin withdrawal treatment/Delusion of inanimate doubles/Delusional depression in 19th-century Scotland/Shoplifting in families of mentally handicapped persons/Photography for anorexia nervosa/ECT mythology?/Manic features during a course of ECT/Philosophy for trainees/Hyperventilation causing asthma/Malignant hyperpyrexia syndrome in combined treatment

\section{The Camberwell Collaborative Depression Study}

SIR: We would like to raise some queries about the third paper in this series reporting a study of depressed patients and their relatives (McGuffin et al, Journal, June 1988, 152, 775-782) which may also be puzzling other readers of the Journal.

Table III purports to test whether life-event associated depression 'breeds true' within families. In order to assess this the probands and their relatives should be compared using the same measure, yet detailed reading of the text does not reassure us that this has been done. First, the period over which life events have been assessed seems to be the three months before onset of depression for the probands but the three months before interview for relatives (pp. 777-778). Given that life events in both patient and relatives are related to onset, this is bound to lead to under-reporting of events for the relatives (the authors note that most of the relatives with depression had experienced onset within 12 months of interview). Second, it is not clear whether Table III uses a different measure of 'life event' for patients from that in Tables I and II. If, as would be expected, the same measure is used, then it is not comparable to that used for relatives (footnote 3 , Table I, and footnote 1 , Table III, make it clear that the focus rating is being used differently). This would again lead to the under-representation of events in relatives. Much of the discussion rests on the assumption that the relationship between events and depression is not as strong in the relatives as in the probands (p. 781), but this is only a legitimate conclusion if comparative measures have been used. Even if comparable measures have been used throughout, it is not clear that the association is stronger among patients than relatives. Since by definition all the patients are depressed, the only way is to look at the percentage with life events. This is roughly $34 \%$ (30/83 in Table I, but $27 / 83$ in Table II, and see also percentages in columns 5 and 6, line 4 of Table VI in the companion paper by Bebbington et al, June 1988, 152, 754-765). The comparable figure for relatives is $50 \%(21 / 42)$. Thus the onset for patients appears, if anything to be less strongly associated with life events than that of the relatives.

Confusions in time periods also arise in Table IV. Here the majority of relatives must have been without depression, but the legend of the table suggests that recent life events occurred in the three months before onset. Perhaps in both Tables III and IV, the authors intended to specify onset for those with ID level of 5 or greater, and three months before interview for the rest. Certainly the figure of 102 relatives with recent life events is common to Tables III and IV, and suggests a common definition.

The figure in Table IV for their community sample subjects with recent life events is surprisingly low given other studies including earlier reports of this sample. A paper published in 1982 based on this community survey gives higher rates for independent events alone during the three months before onset for 'cases' and interview for 'non-cases' (Bebbington et al, 1982). As Tables III and IV include both independent and possibly independent events, we would expect the rates to be higher still. Data from other research in the same area also indicates a higher rate (Brown \& Harris, 1986).

One final puzzle may not be answered by these data, but it is an interesting speculation. Most surveys suggest that about half of those with current depression are suffering from 'chronic' disorders in the sense that onset dates from a time before the last 12 months. It is curious that only $5 \%$ of the relatives 
interviewed in this study did so. Could this unusual feature be in some way related to their being the relatives of depressed probands rather than random community sample cases?

T. K. J. Craig

National Unit for Psychiatric Research and

Development

Lewisham Hospital

London SE13 6LH

UMDS Guy's Campus

London SEI 9RT

Elaine MurPhy

\section{References}

Bebbington, P. E., Tennant, C. \& Hurry, J. (1982) Adversity and the nature of psychiatric disorder in the community. Journal of Affective Disorders, 3, 345-366.

Brown, G. W. \& HARrIS, T. O. (1986) Stressor, vulnerability and depression: a question of replication. Psychological Medicine, 16, 739-744.

SIR: We are grateful to Dr Craig and Professor Murphy for their close reading of our paper and the opportunity to resolve an apparent ambiguity. Their second paragraph is quite correct. In Tables III and IV of our paper, the life events referred to were in the three months before onset for those relatives with an ID level of 5 or greater, and three months before interview for the rest. We hope that this partially relieves their puzzlement referred to in the paragraph, and that we can fully relieve their perplexity by explaining that the same definition of life event was used for both proband and relative in Table III. They should note, however, that we are not making a case that life events have greater impact on probands than their relatives (and we agree that it would be difficult to sustain such an argument on the basis of the data described in our set of papers). The main point is that life events are strongly associated with the onset of depression in a community sample, but not in a sample of first-degree relatives.

The third question relates to the frequency of life events and the comparison we make between the community sample and the first-degree relatives. In our Tables IV and V we use the same definition of life events in both groups. Although we do not provide the results in the paper we did in fact find that threatening life events of any definition were more frequent in relatives than in the community. The apparent discrepancy between the event rate in the community group and that given by Bebbington et al (1981) is due to our use of figures which are weighted back to Bebbington's orginal sample in order to achieve an accurate estimate of the population frequency of recent events. This is necessary because of the two-stage sampling strategy employed by Bebbington et al (1981).

The final point raised by Dr Craig and Professor Murphy is an interesting one, and the question of why there should be a comparatively small proportion of chronic cases among first-degree relatives has also occurred to us. We are inclined toward a more mundane explanation than the one they offer. It seems likely to us that the dating of onset of very broadly defined depression is an inexact procedure and one where we may have adopted a different definition of time of onset from previous workers who have focused on community samples. We used a Past History Schedule (PHS) in conjunction with the Present State Examination (PSE) (McGuffin et al, 1986). The PHS/PSE interviews identify past episodes and define the most severe occurrence if multiple episodes are evident. It may be that this approach more clearly delineates the episodic nature of depression than does a less structured enquiry about past PSE-type symptoms over an extended period.

Department of Psychological Medicine

Peter McGuffiN

University of Wales College of Medicine

Heath Park

Cardiff CF 4 XN

Institute of Psychiatry

Paul Bebbington

London

Department of Psychology

RANDY KaTZ

Toronto General Hospital

\section{References}

Bebbington, P., Henry, J., Tennant, C., et al (1981) Epidemiology of mental disorders in Camberwell. Psychological Medicine, 11, 561-579.

MCGuffin, P., KATZ, R. \& ALDRICH, J. (1986) Past and present state examination: the assessment of 'lifetime ever' psychopathology. Psychological Medicine, 16, 461-466.

\section{Obsessive-compulsive rituals}

SIR: Regarding Katz et al's letter from CIBAGEIGY (Journal, December 1988, 153, 845) about our clomipramine study (Journal, April 1988, 152, 522-534), we can understand that our findings may be unwelcome to them. Their letter contains some confusion and mistakes that need clarification and correction.

In our literature review we wrote "There is no evidence that clomipramine is significantly better than other tricyclic drugs in OCD despite a widely held belief to the contrary." CIBA-GEIGY claim that 